\title{
Using All-Payer Claims Databases to Study Insurance and Health Care Utilization Dynamics
}

\author{
Michael Dworsky, Ph.D. \\ RAND Corporation, Santa Monica, CA, USA.

$\mathrm{C}$ hurning on and off of insurance and across types of private and public insurance are common experiences for American families. ${ }^{1,2}$ Instability in the source of insurance frequently leads to short spells of uninsurance, and prior research shows that insurance instability has adverse consequences for access to primary care. ${ }^{3,4}$ Much of this literature has focused on Medicaid, the Children's Health Insurance Program (CHIP), and other public programs, however, and less is known about how seamless insurance transitions from one insurance carrier to another (those with little or no transitory uninsurance) affect utilization of or access to care.

In this issue of JGIM, Barnett et al. demonstrate how state all-payer claims databases (APCDs) can make it possible to address previously infeasible research questions about the relationship between insurance coverage and health care utilization. ${ }^{5}$ Using data from the linked enrollment and claims files of the Massachusetts APCD, the authors used propensity score matching to compare the trajectories of new physician visits and emergency department (ED) visits between continually insured non-elderly adults who changed insurance carriers ("switchers") and those who remained covered by the same insurance carrier ("non-switchers"). The authors were also able to provide separate estimates for individuals who transitioned from one private insurer to another and for individuals who transitioned from Medicaid to private insurance.

The authors find that insurance switching is a predictor of an immediate spike in visits with new primary care and specialist physicians. They estimate a far larger increase among patients switching from Medicaid to private insurance than among patients transitioning across private insurance carriers. No changes in ED visit frequency were observed among the privately insured; the authors' headline estimates show a slight reduction in ED visits after switches from Medicaid to private coverage, although this finding is less robust than the changes in new physician visits. While this study provides limited detail on the mechanisms that explain these sharp increases in new physician visits, the more pronounced changes for Medicaid enrollees are consistent with the notion that

Published online July 14, 2017 differences across health plans in provider networks drive the observed behavior.

\section{ALL PAYER CLAIMS DATABASES CAN MAKE OTHERWISE IMPOSSIBLE RESEARCH POSSIBLE, BUT NOT WITHOUT SIGNIFICANT EFFORT}

Despite the policy significance of questions about insurance switching and utilization, research on the downstream effects of insurance switching has been limited in part by the formidable data requirements of such a study. Indeed, this study's innovation is as much methodological as it is substantive. Health policy research using APCDs has blossomed in recent years, ${ }^{6}$ but studies that leverage APCD enrollment and claims files simultaneously have been slower to emerge. To understand why, readers should consult the appendix that the authors provide, which very nicely details the substantial effort they had to invest in data cleaning and merging of additional information such as physician specialties.

A particularly thorny obstacle to studying transitions across payers is the fact that the Massachusetts APCD does not collect Social Security numbers or other unique person identifiers. The possibility of errors in linking records longitudinally is a serious threat for research on insurance transitions: this could introduce measurement error that is a direct consequence of insurance transitions. Barnett et al. were careful to demonstrate that their findings were robust among the minority of claims with highconfidence matches. This is just one of the ways in which the authors demonstrate excellent craftsmanship in handling a complex and messy database, and this study should provide a useful road map for future efforts to examine the joint dynamics of insurance coverage and health care utilization using APCD data.

\section{APCDs Are Not a Silver Bullet for Causal Inference, But Creative Researchers Will Find a Way}

Despite the use of propensity score matching to balance selected characteristics between switchers and non-switchers, the authors' ability to draw causal inferences from this research design is limited, because some insurance transitions may reflect unobserved changes in life circumstances or health care needs that could lead to endogeneity between changes in health care utilization and changes in insurance coverage. The risk of 
endogeneity may be quite acute here because pre-switching health care utilization is not used in the matching process.

To definitively answer health policy questions, causal inference is often necessary. As with all administrative data, researchers must weigh the completeness of APCD data against potential limitations in terms of individual characteristics (e.g., family income) or contextual variables (e.g., premiums and other information about the menu of insurance plans available to an individual) that one might like to use in designing a quasiexperimental study.

When causal inference is critical, creative researchers will often be able to devise strategies that make clever use of the APCD's strengths to work around its weaknesses. In the appendix to the present study, for instance, the authors are able to define a subset of plausibly exogenous insurance switches by identifying group health plans that lose an abnormally high number of enrollees at once - a useful strategy for complete administrative data that recalls the analysis of displaced workers in labor economics. ${ }^{7}$

\section{What Are the Next Questions Raised by this Work?}

This work raises questions that should be examined in future research with the Massachusetts APCD; many of these questions might also be amenable to study in other states as APCDs elsewhere mature.

One question is whether the short-term effects observed here are transitory or persistent. The graphical evidence suggests that utilization by insurance switchers converges toward the levels observed among non-switchers, but the 12-month follow-up period available here does not support definitive conclusions. Longer panels of APCD data (including updates of the data used in this study) have the potential to capture these longer-term effects. Longer-term effects on new physician visits may be of limited interest, but broader measures of utilization or those that reflect patient outcomes would warrant examination.

A different direction for research could be taken that would help assess the policy implications of this study's findings - examining whether there are meaningful differences across individuals in the probability of switching insurance coverage. One might imagine that insurance switching is correlated across policy years, and that a subset of individual market consumers account for a disproportionate share of insurance switches over time. Indeed, the propensity for switching could be unevenly distributed for a variety of reasons that have different policy implications. Are insurance switchers more medically or financially vulnerable than other policyholders to changes in providers associated with switching? This could be the case if low-income consumers were more price-sensitive or those with chronic conditions were more likely to switch due to changes in cost-sharing, formularies, or other plan attributes. Yet one might just as well imagine that switching is concentrated among healthier or more financially literate consumers. The composition of the switcher population may also have implications for the benefit design incentives facing insurers.
Questions about variation in individual propensities for switching insurance have remained largely unexplored due to the somewhat limited sample sizes and relatively short panel dimension of non-administrative data sources typically used for studying insurance transitions. If states are able to negotiate the challenges posed by the Supreme Court's decision in Gobeille v. Liberty Mutual (2015), which allows self-insured plans to opt out of APCDs, ${ }^{8}$ APCDs should increasingly facilitate novel research on longer-term trajectories of insurer switching.

\section{Insurer Switching Will Remain Important With or Without the Affordable Care Act}

The Affordable Care Act (ACA) has led to dramatic growth in the number of Americans covered by non-group private insurance. The most recent data show that 10.3 million individuals had marketplace policies in effect as of February. ${ }^{9}$ Although the future of the ACA is uncertain, the American Health Care Act of 2017 - the repeal-and-replace legislation passed by the House of Representatives - would continue the ACA's reliance on a subsidized non-group health insurance market to make coverage available to individuals ineligible for Medicaid and not covered by employer-sponsored coverage. Despite the current legislative uncertainty, it seems safe to say that the consequences of insurance transitions are a topic that will continue to grow in importance with or without the ACA. APCDs should have a central role to play in this research agenda, and the methods demonstrated here will be an important starting point for future researchers.

Corresponding Author: Michael Dworsky, Ph.D.; RAND Corporation, Santa Monica, CA, USA (e-mail: mdworsky@rand.org).

Conflict of Interest: The author declares that he does not have a conflict of interest.

\section{REFERENCES}

1. Short PF, Graefe DR. Battery-powered health insurance? Stability in coverage of the uninsured. Health Aff 2003;22(6):244-55.

2. Short PF, Graefe DR, Swartz $\mathbf{K}$, Uberoi N. New estimates of gaps and transitions in health insurance. Med Care Res Rev [Internet] 2012;69(6):72136. Available from: http://www.ncbi.nlm.nih.gov/pubmed/22833452

3. Orzol SM, Hula L, Harrington M. Program churning and transfers between medicaid and CHIP. Acad Pediatr [Internet] 2015;15(3):S56-63. doi: 10.1016/j.acap.2015.02.006.

4. Buchmueller T, Orzol SM, Shore-Sheppard L. Stability of children's insurance coverage and implications for access to care: Evidence from the Survey of Income and Program Participation. Int J Health Care Finance Econ 2014;14(2): 109-26.

5. Barnett ML, Song Z $\mathbf{Z}$, Rose S, et al. Insurance Transitions and Changes in Physician and Emergency Department Utilization: An Observational Study. J Gen Intern Med. 2017 doi: 10.1007/s11606-017-4072-4.

6. Ericson KM, Starc A. Measuring consumer valuation of limited provider networks. Am Econ Rev 2015;105(5):115-9.

7. Jacobson LS, LaLonde RJ, Sullivan DG. Earnings Losses of Displaced Workers. Am Econ Rev 1993;83(4):685-709.

8. Freedman JD, Green L, Landon BE. All-Payer Claims Databases - Uses and Expanded Prospects after Gobeille. N Engl J Med [Internet] 2016;375(23):22157. Available from: http://www.nejm.org/doi/10.1056/NEJMp1609578

9. Centers for Medicare and Medicaid Services. 2017 Effectuated Enrollment Snapshot [Internet]. 2017;(June):1-15. Available from: https://downloads. cms.gov/files/effectuated-enrollment-snapshot-report-06-12-17.pdf 